\title{
Implementación del modelo de gestión estratégico para la mejora de la calidad en la cadena de suministros
}

\section{Implementation of the strategic management model for the improvement of quality in the supply chain}

\author{
LÓPEZ-GARZA, Esmeralda $\dagger^{*}$, FUENTES-RUBIO, Yadira Aracely, DOMÍNGUEZ-CRUZ, René \\ Fernando y MÉNDEZ-PÉREZ Aldo Luis
}

Centro de Innovación Tecnológica en Eléctrica y Electrónica. Universidad Autónoma de Tamaulipas

ID $1^{\mathrm{er}}$ Autor: Esmeralda, López-Garza / ORC ID: 0000-0002-6463-7946, CVU CONACYT ID: 637087

ID $1^{\mathrm{er}}$ Coautor: Yadira Aracely, Fuentes-Rubio / ORC ID: 0000-0002-7385-9794, CVU CONACYT ID: 794463

ID $2^{\text {do }}$ Coautor: René Fernando, Domínguez-Cruz / ORC ID: 0001-7001-7543, Researcher ID Thomson: C-3108-2018, CVU CONACYT ID: 31057

ID $3^{\text {er }}$ Coautor: Aldo Luis, Méndez-Pérez / ORC ID: 0003-1546-7562, CVU CONACYT ID: 121670

DOI: $10.35429 /$ JED.2019.19.6.18.23

Recibido: 15 de Abril de 2019; Aceptado: 20 de mayo de 2019

\begin{abstract}
Resumen
El objetivo de este artículo es mostrar los resultados obtenidos en el diseño e implementación de un modelo de gestión estratégico basado en la metodología de Balanced Scorecard (BSC) y aplicado a una empresa del sector automotriz el cual le permita poseer una mayor competitividad. La metodología desarrollada permitió hacer una revisión de la visión, misión y objetivos de la empresa con la finalidad de alinear a los objetivos del departamento de calidad de proveedores, de tal manera que los indicadores obtenidos impacten directamente en los KPI de la compañía y ello genere un mapa estratégico para el desarrollo de proveedores. Dichos indicadores permiten medir el desempeño de las empresas, estableciendo métricos con mayor impacto, los cuales posee una gran importancia a nivel mundial. El BSC está ligado con los Sistemas de Producción Toyota (TPS), donde uno de sus pilares es el Justo a tiempo y por ello la importancia de disponer un métrico OTD (Cumplimiento de entregas). Con su implementación, se obtuvo los siguientes indicadores para evaluar a los proveedores: Material Defectuoso, OTD, Proceso de Aprobación de Partes de Producción y Defectos por Millón. Estos resultados muestran una mejorar en la eficiencia en la empresa y de los proveedores debido a que impactaron directamente en los métricos KPI, OTD y CONC (Costo de No-Conformidad).
\end{abstract}

\begin{abstract}
The aim of this paper is to show the results about the design and implementation of a strategic management model based on the Balanced Scorecard (BSC) methodology applied to a company in the automotive activity which allows it to have greater competitiveness. The developed methodology allowed a review of the vision, mission and objectives of the company in order to align the objectives of the supplier quality department, in such a way that the indicators obtained directly impact the KPI of the company and this generates a strategic map for the development of suppliers. These indicators allow measuring the performance of companies, establishing metrics with greater impact, which is of great importance in worldwide. The BSC is linked to the Toyota Production Systems (TPS), where one of its pillars is the Just in Time and therefore the importance of having an OTD metric (Delivery Compliance). The implementation of this methodology, we obtained the following indicator to evaluate the suppliers: Defective Material, OTD, Process of Approval of Production Parts and Defects per Million. These results allowed improving the efficiency in the company and that of the suppliers because they directly impacted the KPI, OTD and CONC metrics.
\end{abstract}

Calidad, Balanced Scorecard, Desarrollo de Proveedores

Quality, Balanced Scorecard, Supplier Development

Citación: LÓPEZ-GARZA, Esmeralda, FUENTES-RUBIO, Yadira Aracely, DOMÍNGUEZ-CRUZ, René Fernando y MÉNDEZ-PÉREZ Aldo Luis. Implementación del modelo de gestión estratégico para la mejora de la calidad en la cadena de suministros. Revista de Desarrollo Económico. 2019, 6-19. 18-23

\footnotetext{
*Correspondencia al Autor (Correo Electrónico: elgarza@ docentes.uat.edu.mx)

$\dagger$ Investigador contribuyendo como primer autor.
} 


\section{Introducción}

En el mundo industrial, la competitividad es un factor fundamental para el crecimiento de una empresa manufacturera, debido a que constantemente compiten para captar mercados y recursos; lo cual ha llevado a implementar metodologías que ayuden a evaluar el desempeño de las empresas (Huerta et al 2018). Por ello un factor clave es la medición adecuada de la competitividad de la industria, mediante la evolución de indicadores claves de desempeño, el cual requiere de un diagnostico estratégico encaminado a identificar su posición competitiva, fortalezas y debilidades. (Smith 2016).

Una de las formas para aumentar la competitividad de una empresa es mediante los sistemas de gestión de calidad, viéndose reflejado en la reputación de la empresa, e imagen de la marca, todas procedentes de la mejora continua destinadas para incrementar la satisfacción del cliente.

Por lo tanto, se puede establecer que la calidad es una fuente de ventaja competitiva como lo menciona Hereas et al (2009), que genera resultados económicos-financieros de la empresa, debido a la disminución de los costos generados por no calidad.

En los sistemas de mejora de la calidad, los sistemas productivos TPS, debido a su filosofía Justo a tiempo aseguran una operativa en el flujo continuo y regular, de esta manera si el sistema productivo se mantiene en funcionamiento correcto se aseguran las entregas a tiempo a los clientes asegurando de esta manera la satisfacción de los clientes (Cuatrecasas 2017).

Dichos sistemas se pueden aplicar en cualquier parte de la cadena de suministros, por ejemplo, en el proceso de materia prima, en donde si no cuenta con la calidad necesaria impacta directamente en el producto final.

Por ello es importante un desarrollo correcto de los proveedores en donde se puedan evaluar correctamente y se establezca, un acompañamiento para la implementación de estrategias que ayuden a mantener su calidad y entregas a tiempo. Así de esta manera impacta directamente en la satisfacción del cliente de la empresa.
El BSC es una metodología estratégica de diagnóstico, que ayuda a monitorear y evaluar los indicadores claves de la empresa con claridad, con la finalidad de implementar estrategias de mejora para lograr el cumplimento de las metas. (Amo Baraybar, 2010)

En este trabajo se llevó a cabo la implementación de un BSC dentro de una empresa de giro automotriz, concretamente en el área desarrollo de proveedores de calidad. La selección de área se centra en la problemática en la materia prima que le suministra, debido a los rechazos de calidad en la línea de producción y quejas de los clientes. Esto a consecuencia de la calidad de la materia prima, y el incumplimiento con las fechas de entrega, por ello este estudio tiene como objetivo principal seleccionar los indicadores para medir el desempeño de los proveedores de una manera eficiente.

Adicionalmente, con la estrategia implementada se logró identificar aquellos proveedores que no cumplen con los requerimientos de producción para la implementación de estrategias logrando aumentar un $18 \%$ y disminuir un 5\% los costos por no calidad.

\section{Balanced Scorecard}

Según Scaramussa (2010), el BSC es un sistema completo de gerencia que permite la integración tanto de aspectos del direccionamiento estratégico, como la misma evaluación de desempeño que ha tenido el negocio.

Esta planeación estratégica integradora de los diferentes aspectos de la empresa, representa una revolución con relación al tradicional enfoque gerencial, es decir, asumir el autocontrol. De esta manera permite tener el control del estado de salud corporativa y la forma cómo se están encaminando las acciones para alcanzar la visión.

A partir de la visualización y el análisis de los indicadores balanceados, pueden tomarse acciones preventivas o correctivas que afecten el desempeño global de la empresa (Sánchez 2010). Esta estrategia consta de cuatro perspectivas de acuerdo con Morales et al (2007): 
Cliente: Es preciso que los gerentes den un giro y traduzcan la misión organizacional con respecto a los clientes y para lograrlo es necesario establecer un objetivo claro de dirección, al igual que una definición precisa de los indicadores de desempeño. En este punto deben establecerse objetivos según los requerimientos de los clientes, los cuales varían según el tipo de organización y mercado.

Negocio interno: Hace referencia a la perspectiva interna que compone la excelencia que debe caracterizar a cada uno de los departamentos de la organización, pues lo principal es que haya un enfoque de operaciones internas críticas que permitan satisfacer a los clientes.

Innovación y aprendizaje: Plantea la importancia que representa para la empresa incluir dentro de sus valores y formas de medición, la capacidad de mejora con el tiempo, puesto que es la única fórmula aseguradora de éxito.

Perspectiva financiera: Reflejan la capacidad de capitalización de los logros obtenidos y la conversión de éstos en ganancias para la empresa. El desafío organizacional está en alinear de la mejor manera las operaciones y finanzas con el propósito de proyectar las mejoras y desarrollar una planificación de estrategias de la mejor manera posible.

Estas perspectivas abarcan todos los procesos necesarios para el correcto funcionamiento de una empresa y deben ser considerados en la definición de los indicadores.

El primer paso para la implementación del BSC según Kaplan et al. (2009) es la definición de la visión y estrategias, entendida la visión y estrategias de la empresa es posible determinar los objetivos que hay que cumplir para lograr la estrategia y aterrizarlos en indicadores, estos deben reflejar los resultados muy puntuales de los objetivos, pero también deberán informar sobre el avance para alcanzarlos.

Una vez definido el modelo de negocio y los indicadores de acción y resultados, es posible implementar el BSC de acuerdo con Pérez rivera, E. J. (2010) de la siguiente manera:
Modelo de control y seguimiento. En caso de que la visión, estrategias e indicadores estén perfectamente definidos y acordados, el BSC puede implementarse como un modelo de análisis por excepción. Se da un seguimiento puntual sobre los avances en el logro de las estrategias con respecto a lo planteado y el BSC libera una cantidad de trabajo importante al directivo, al realizar análisis por excepción de aquellos procesos conocidos que, eventualmente, requieren de más tiempo para su análisis; un análisis que sólo se da cuando no corresponden los datos con el objetivo. De esta manera el BSC mantiene un proceso permanente en el que puede haber una retroalimentación de un ciclo, que consiste en corregir las desviaciones para alcanzar los objetivos fijos definidos. La retroalimentación sugiere aprendizaje estratégico, que es la capacidad de formación de la organización a nivel ejecutivo, es el poder aprender la forma de utilizar como un sistema de gestión estratégica.

\section{Metodología}

Para la aplicación del BSC de acuerdo con la metodología establecida de Membrado (2013) es necesaria la definición de la misión, visión y valores de la organización. En la Figura 1, muestra las estrategias de la empresa mediante las 4 perspectivas, Financiera, Cliente, Interna y Aprendizaje y Crecimiento alineadas con la visión, valores y misión de la empresa. De esta manera se puede establecer que las metas del departamento de desarrollo de proveedores estén a las de las metas y así poder definir correctamente los indicadores adecuados para la evaluación.

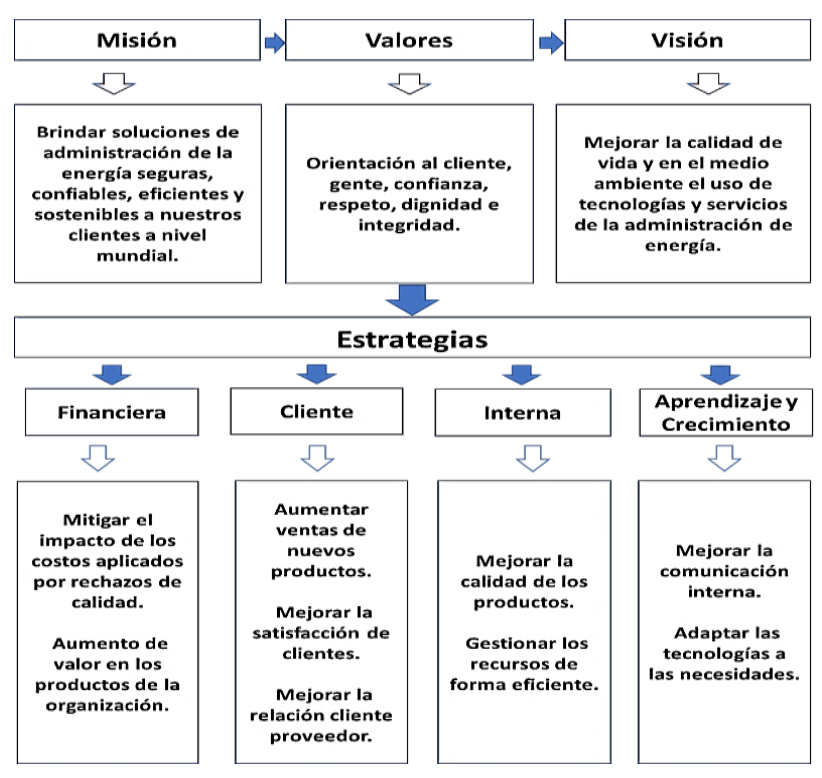

Figura 1 Mapa Estratégico

Fuente: Elaboración Propia

LÓPEZ-GARZA, Esmeralda, FUENTES-RUBIO, Yadira Aracely, DOMÍNGUEZ-CRUZ, René Fernando y MÉNDEZ-PÉREZ Aldo Luis. Implementación del modelo de gestión estratégico para la mejora de la calidad en la cadena de suministros. Revista de Desarrollo Económico. 2019 
Una vez establecido ella selección de los indicadores a medir los cuales impactan directamente en el desempeño de los proveedores como son:

DMR (por sus siglas en inglés Defective Material Report, reporte de material defectuoso). En donde para evaluar al proveedor se contabilizaron cuantos DMR incurrieron en un periodo y poder cuantificar como se muestra en la Tabla 1. Si no incurre a un DMR se le otorga 10 pts., de 1 a 5 DMR en el periodo de tiempo obtiene 5 pts., y si son más de 5 no obtiene puntos.

\begin{tabular}{|l|r|}
\multicolumn{1}{|c|}{ DMR } \\
\multicolumn{1}{|c|}{ Rangos de puntaje } & Puntos \\
\hline 0 DMR & 10 \\
\hline $1-5$ DMR & 5 \\
\hline Mas de 5 & 0 \\
\hline
\end{tabular}

Tabla 1 Evaluación del desempeño del proveedor mediante el Indicador DMR

Fuente: Elaboración Propia

\section{- DMR Containment Response (Respuesta de contención en menos de 48hrs), al ser generado un DMR a proveedor se evalúa que las acciones de contención se generen en forma y un tiempo menor de $48 \mathrm{hrs}$. La forma para evaluar a los proveedores se ve reflejado en la Tabla 2, en donde si responden en un porcentaje de $90 \%$ a $100 \%$ en tiempo obtiene 10 pts., y en menos del $49 \% 0$ pts.}

\begin{tabular}{|l|r|}
\hline \multicolumn{2}{|c|}{$\begin{array}{c}\text { DMR Containment response } \\
\text { Rangos de puntaje }\end{array}$} \\
\hline 90\% a 100\% de los DMR en tiempo & 10 \\
\hline $50 \%$ a 89\% en responder a tiempo & 5 \\
\hline Menos de 49\% en responder a tiempo & 0 \\
\hline
\end{tabular}

Tabla 2 Evaluación del desempeño del proveedor mediante el Indicador DMR Containment response Fuente: Elaboración Propia

OTD. Este indicador mide el cumplimiento de entregas de material en tiempo y forma de los proveedores. Los proveedores que obtengan un cumplimiento del $95 \%$ al $100 \%$ son aquellos que en su desempeño se les otorga 40pts, tal como se muestra en la Tabla 3 donde señala el puntaje correspondiente de acuerdo con el \% obtenido.

\begin{tabular}{|l|c|}
\multicolumn{1}{|c|}{ OTD } \\
Rangos de puntaje & Puntos \\
\hline $100 \%-95 \%$ & 40 \\
\hline $94 \%-80 \%$ & 30 \\
\hline $79 \%-60 \%$ & 20 \\
\hline $59 \%-30 \%$ & 10 \\
\hline Menor de $29 \%$ & 0 \\
\hline
\end{tabular}

Tabla 3 Evaluación del desempeño del proveedor mediante el Indicador OTD

- PPAP Rejected (por sus siglas en inglés Production Part Approval Process, y su significado proceso de aprobación de partes de producción), indicador que medirá los rechazos en la evaluación de nuevos productos. En la tabla 4 se refleja los puntos que recibe el proveedor de acuerdo con los rechazos obtenidos en el ciclo.

\begin{tabular}{|l|r|}
\hline \multicolumn{1}{|c|}{ PPAP Rechazos } \\
Rango de puntaje & Puntos \\
\hline 1-2 Rechazos & 10 \\
\hline Mas de 3 Rechazos & 5 \\
\hline
\end{tabular}

Tabla 4 Evaluación del desempeño del proveedor mediante el Indicador PPAP Rejected

- $\quad$ PPM External P1 y P2 (por sus siglas en inglés Parts Per Million, y su significado partes por millón), indicador que medirá DPPM para proveedores externos, los cuales son todos aquellos que no son de la empresa se asignan de acuerdo con la meta establecida para cada área. En la tabla 5 muestra la puntuación que obtienen los proveedores externos de la planta 1 , en la tabla 6 muestra la puntuación para los proveedores externos de la planta 2.

\begin{tabular}{|l|r|}
\hline \multicolumn{2}{|c|}{ PPM External P1 } \\
Rangos de puntos & Puntos \\
\hline $0-1047$ & 30 \\
\hline $1048-2096$ & 20 \\
\hline $2097-3144$ & 10 \\
\hline Mas de 3145 & 0 \\
\hline
\end{tabular}

Tabla 5 Evaluación del desempeño del proveedor externos de la planta 1, mediante el Indicador PPM

\begin{tabular}{|l|r|}
\hline \multicolumn{2}{|c|}{ PPM External P2 } \\
Rangos de puntos & \multicolumn{1}{c|}{ Puntos } \\
\hline $0-914$ & 30 \\
\hline $915-1829$ & 20 \\
\hline $1830-2744$ & 10 \\
\hline Mas de 2745 & 0 \\
\hline
\end{tabular}

Tabla 6 Evaluación del desempeño del proveedor externos de la planta 2, mediante el Indicador PPM

LÓPEZ-GARZA, Esmeralda, FUENTES-RUBIO, Yadira Aracely, DOMÍNGUEZ-CRUZ, René Fernando y MÉNDEZ-PÉREZ Aldo Luis. Implementación del modelo de gestión estratégico para la mejora de la calidad en la cadena de suministros. Revista de Desarrollo Económico. 2019 
- PPM Internal, indicador que medirá DPPM para proveedores internos, los cuales son plantas de la misma empresa que abastecen material como proveedor, se asignan de acuerdo con la meta establecida para cada planta como se observa en la tabla 7 para planta $1 \mathrm{y}$ tabla 8 para planta 2 .

\begin{tabular}{|l|c|}
\hline \multicolumn{2}{|c|}{ PPM Internal P1 } \\
Rangos de puntos & Puntos \\
\hline $0-1822$ & 30 \\
\hline $1823-3645$ & 20 \\
\hline $3646-5468$ & 10 \\
\hline Mas de 5469 & 0 \\
\hline
\end{tabular}

Tabla 7 Evaluación del desempeño del proveedor internos de la planta 1, mediante el Indicador PPM

\begin{tabular}{|l|c|}
\hline \multicolumn{2}{|c|}{ PPM Internal Control } \\
Rangos de puntos & Puntos \\
\hline $0-118$ & 30 \\
\hline $119-238$ & 20 \\
\hline $239-357$ & 10 \\
\hline Mas de 358 & 0 \\
\hline
\end{tabular}

Tabla 8 Evaluación del desempeño del proveedor internos de la planta 2, mediante el Indicador PPM

Los rangos establecidos para medir el desempeño general de los proveedores son los siguientes:

\begin{tabular}{|l|c|}
\hline \multicolumn{1}{|c|}{ Puntaje Total } & Evaluación \\
\hline $100-90$ & Preferente \\
\hline $89-70$ & Aprobado \\
\hline $69-0$ & Probatorio \\
\hline
\end{tabular}

Tabla 9 Rangos establecidos para la evaluación de proveedores

\section{Resultados}

Al implementar la metodología del BSC basada en el esquema descritos en la Figura 1 y Tablas 1-9 en la que se obtiene el siguiente cuadro de mando Tabla 10, donde se indica a los proveedores que al momento de evaluarlos obtuvieron una calificación probatoria.

En la Parte (a) y (b) de la Tabla 10 el cual se describe de izquierda a derecha, definiendo proveedores internos y externos, seguido del código el área al que pertenece, sea Planta 1 (P1) o Planta $2(\mathrm{P} 2)$ y el número de parte $\mathrm{y}$ proveedor que se le asigno y los indicadores previamente ya definidos, en los cuales vemos el puntaje obtenido de acuerdo en su desempeño en cada rubro, acompañada del puntaje correspondiente en el indicador.
En la parte (c) de la Tabla 9 se obtiene la calificación por proveedor sumando todos los puntos que obtuvieron en los indicadores, categorizándolos de menor a mayor eficiencia, donde el color rojo los coloca en prueba.

\begin{tabular}{|l|l|r|r|r|r|r|}
\hline \multicolumn{2}{c}{ P } & \# P y P & PPM & Pts. & DMR & Pts. \\
\hline E & P1 & 1 & 4501 & 0 & 32 & 0 \\
\hline E & P2 & 2 & 1000 & 0 & 9 & 0 \\
\hline I & P1 & 3 & 4854 & 10 & 13 & 0 \\
\hline E & P1 & 4 & 5412 & 0 & 3 & 5 \\
\hline I & P2 & 5 & 250 & 20 & 34 & 0 \\
\hline E & P2 & 6 & 59 & 0 & 1 & 5 \\
\hline E & P1 & 7 & 2892 & 10 & 11 & 0 \\
\hline E & P2 & 8 & 2 & 30 & 1 & 5 \\
\hline \multicolumn{7}{|c|}{ Parte (a) } \\
\hline
\end{tabular}

\begin{tabular}{|l|l|r|r|r|r|r|r|r|r|}
\hline & \multicolumn{1}{l}{$\begin{array}{l}\text { \# P } \\
\text { DMR }\end{array}$} & \multicolumn{1}{l}{ Pts. } & OTD & Pts. & PPAP & Pts. \\
\hline E & P1 & 1 & $2 \%$ & 0 & 77 & 20 & 0 & 10 \\
\hline E & P2 & 2 & $25 \%$ & 0 & 84 & 30 & 0 & 10 \\
\hline I & P1 & 3 & 0 & 0 & 92 & 30 & 0 & 10 \\
\hline E & P1 & 4 & 0 & 0 & 100 & 40 & 0 & 10 \\
\hline I & P2 & 5 & $36 \%$ & 0 & 82 & 30 & 2 & 5 \\
\hline E & P2 & 6 & 0 & 0 & 99 & 40 & 0 & 10 \\
\hline E & P1 & 7 & 0 & 0 & 98 & 40 & 0 & 10 \\
\hline E & P2 & 8 & 0 & 0 & 79 & 20 & 0 & 10 \\
\hline
\end{tabular}

\begin{tabular}{|l|l|r|r|}
\hline \multicolumn{2}{|c|}{ P } & \# P y P & Puntaje Total \\
\hline E & P1 & 1 & $\mathbf{3 0}$ \\
\hline E & P2 & 2 & $\mathbf{4 0}$ \\
\hline I & P1 & 3 & $\mathbf{5 0}$ \\
\hline E & P1 & 4 & $\mathbf{5 5}$ \\
\hline I & P2 & 5 & $\mathbf{5 5}$ \\
\hline E & P2 & 6 & $\mathbf{5 5}$ \\
\hline E & P1 & 7 & $\mathbf{6 0}$ \\
\hline E & P2 & 8 & $\mathbf{6 5}$ \\
\hline
\end{tabular}

Parte (c)

Tabla 10 Puntajes obtenidos al evaluar los proveedores de la empresa. En la parte (a) muestra los indicadores PPM y DMR para los proveedores con su respectivo puntaje. En la parte (b) muestra los resultados en los indicadores DMR ORT, OTD, PPAP, con sus correspondientes resultados. En la parte (c) corresponde a la sumatoria total de los puntos obteniendo la calificación final del proveedor.

De acuerdo con el desempeño de la Tabla 10 al inicio del uso del BSC, fueron seleccionados los proveedores impactantes $\mathrm{O}$ con menor puntaje, teniendo un total de ocho proveedores, cuatro para el área de P1 y cuatro para el área de P2.

Una vez informados los proveedores impactantes, se iniciaron las actividades previamente definidas para el Ingeniero de Calidad. 
Programando revisiones semanales por medio de conferencias telefónicas, definiendo las acciones y las fechas de vencimiento de cada una de ellas; dichas acciones fueron establecidas de acuerdo con cada problema de calidad, utilizando la metodología de 8D el cual de acuerdo con Izaguirre (20017) consiste en 8 Disciplinas, las cuales son las siguientes:

\section{- D1: Formar un equipo \\ - D2: Definir el problema \\ - D3: Implementar acciones de contención \\ - D4: Identificar y verificar la causa raíz \\ - D5: Determinar acciones correctivas permanentes \\ - D6: Implementar y verificar las acciones \\ - correctivas permanentes \\ - D7: Prevenir la recurrencia del problema y/o su causa raíz}

- $\quad$ D8: Reconocer los esfuerzos del equipo

Esto con la finalidad de mejorar el desempeño en DPPM y a su vez con estas acciones lograr reducir la cantidad de DMR generados y la respuesta en acciones de contención. De igual manera se establecieron acciones para aumentar las entregas a tiempo y los PPAP rechazados. A continuación, se muestran los resultados de acuerdo con el BCS (Tabla 11) en el siguiente periodo después de realizar las estrategias de mejora, los proveedores impactantes obtuvieron una mejora en su desempeño, reduciendo la cantidad de DPPM y aumentando el porcentaje de entregas a tiempo.

\begin{tabular}{|l|l|r|r|r|r|r|}
\hline \multicolumn{2}{|c}{ P } & \# P y P & PPM & \multicolumn{1}{c|}{ Pts. } & \multicolumn{1}{c|}{ DMR } & Pts. \\
\hline E & P1 & 7 & 1039 & 20 & 3 & 5 \\
\hline I & P1 & 3 & 440 & 30 & 1 & 5 \\
\hline E & P1 & 1 & 1172 & 20 & 5 & 5 \\
\hline E & P1 & 4 & 198 & 30 & 2 & 5 \\
\hline I & P2 & 5 & 295 & 30 & 10 & 0 \\
\hline E & P2 & 6 & - & 30 & 0 & 10 \\
\hline E & P2 & 2 & 300 & 30 & 1 & 5 \\
\hline E & P2 & 8 & - & 30 & 0 & 10 \\
\hline
\end{tabular}

\begin{tabular}{|l|l|r|r|r|r|r|r|r|}
\hline \multicolumn{10}{|c|}{\begin{tabular}{l} 
Parte (a) \\
\hline
\end{tabular}} & P & \multicolumn{1}{|l|}{$\begin{array}{l}\text { DMR } \\
\text { ORT }\end{array}$} & \multicolumn{1}{l|}{ Pts } & \multicolumn{1}{l|}{ OT } & \multicolumn{1}{l|}{ Pts } & \multicolumn{2}{l|}{ PPA } & \multicolumn{1}{l|}{ Pts } \\
\hline E & P1 & 7 & $100 \%$ & 10 & 79 & 20 & 0 & 10 \\
\hline I & P1 & 3 & 0 & 0 & 90 & 30 & 0 & 10 \\
\hline E & P1 & 1 & $90 \%$ & 10 & 85 & 30 & 0 & 10 \\
\hline E & P1 & 4 & $50 \%$ & 5 & 83 & 30 & 0 & 10 \\
\hline I & P2 & 5 & $100 \%$ & 10 & 84 & 30 & 0 & 10 \\
\hline E & P2 & 6 & $100 \%$ & 10 & 92 & 30 & 0 & 10 \\
\hline E & P2 & 2 & $100 \%$ & 10 & 95 & 40 & 0 & 10 \\
\hline E & P2 & 8 & $100 \%$ & 10 & 100 & 40 & 0 & 10 \\
\hline
\end{tabular}

Parte (b)

\begin{tabular}{|l|r|r|r|}
\hline \multicolumn{2}{|c}{ P } & \# P y P & \multicolumn{1}{c|}{$\begin{array}{c}\text { Puntaje } \\
\text { Total }\end{array}$} \\
\hline E & P1 & 7 & 65 \\
\hline I & P1 & 3 & 75 \\
\hline E & P1 & 3 & 75 \\
\hline E & P1 & 1 & 75 \\
\hline I & P2 & 4 & 80 \\
\hline E & P2 & 5 & 80 \\
\hline E & P2 & 6 & 90 \\
\hline E & P2 & 2 & 95 \\
\hline E & P1 & 8 & 100 \\
\hline \multicolumn{3}{|c|}{ Parte (c) }
\end{tabular}

Tabla 11 Puntajes obtenidos al evaluar los proveedores después de las acciones correctivas de la empresa. En la parte (a) muestra los indicadores PPM y DMR para los proveedores con su respectivo puntaje. En la parte (b) muestra los resultados en los indicadores DMR ORT, OTD, PPAP con sus correspondientes resultados. En la parte (c)

\section{Conclusión}

El modelo BSC se presenta como un método en donde se puede centralizar la gestión estratégica, involucrando a todas las áreas de la organización, pero con un enfoque especifico en 2 de los KPI como es el OTD aumentando las entregas a tiempo en un $18 \%$ y el CONC disminuyendo los costos de no conformidad en un 5\% tal como se muestra en el Gráfico 1.

\section{Comportamiento de los KPI de la empresa} antes y despues del BSC

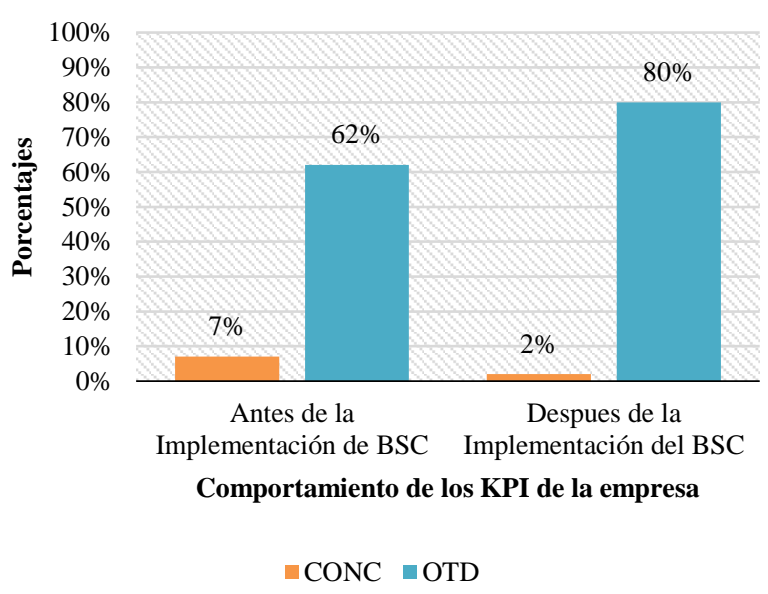

Gráfico 1 Comportamiento de los KPI de la empresa antes y después de la implementación del BSC Fuente: Realización Propia

Los aportes que deja la elaboración de este modelo, es la evidencia de los cambios positivos vistos con el uso de esta herramienta, los cuales de observan en los indicadores PPM, DMR, DMR ORT, OTD y PPAP. 
La disminución de los rechazos de calidad en los productos, el aumento de las entregas a tiempo y la respuesta en tiempo y forma de los proveedores, son los resultados del trabajo y seguimiento a las acciones realizadas mes a mes. Por lo anterior, se considera que la implementación de la estrategia sugerida fue exitosa.

\section{Recomendaciones}

Debido a los beneficios que aportó esta metodología, se recomienda establecer este método permanentemente en la empresa, de esta manera se puede visualizar aquellos proveedores que requieren de estrategias y se trabaje en conjunto para su desarrollo. Al igual ayuda a detectar esos proveedores preferentes para felicitarlos y exhortarlos a que continúen realizando su trabajo correctamente. Esto trae consigo una estrecha comunicación para beneficio de ambas partes.

\section{References}

Huerta-Dueñas, Sandoval (2018). Sistemas de Calidad como Estrategia de Ventaja Competitiva.

Smith Ramírez (2016) El impacto de la estrategia de calidad en el desempeño de la organización. Revista Ciencias Estratégicas, vol. 24 , núm. 35 .

Hereas, Arana, Camisón, Casadesus, Martiarena (2008). Gestión de la calidad y la competitividad de las empresas de la CAPV. Universidad de Deusto. Bilbao.

Cuatrecasas, Babón (2017). Gestión integral de la Calidad. Implantación, control y certificación. Editorial PROFIT

Amo Baraybar, F., (2010). El Cuadro de Mando Integral «Balanced Scorecard». España: Esic.

Scaramussa, S. et al. (2010). La contribución del Balanced Scorecard como instrumento de gestión estratégica en el apoyo a la gerencia". Revista Visión de Futuro, Volumen 13, Año 7, $\mathrm{N}^{\mathrm{o}} 1$.

Sánchez Córdoba (2010). El Balanced Scorecard como herramienta de gestión en las organizaciones del siglo XXI. Revista Gestión y Desarrollo. Volumen 7. No. 2.
Morales Souquett, Pinilla (2007). Balanced scorecard como herramienta de diagnóstico Visión Gerencial, núm. 1, pp. 82-92.

Kaplan, R. et al. (2009). Cuadro de manto integral: The Balanced Scorecard. Barcelona, Gestión 2000. p. 124.

Kaplan, Robert S., and Norton, David P. (2000), Having Trouble With Your Strategy? Then Map It", Harvard Business Review.

Kaplan, Robert S., and Norton, David P. (1996), "The Balanced Scorecard: Translating Strategy into Action", Harvard Business School Press.

Kaplan, Robert S., and Norton, David P. (2001), "The Strategy Focused Organization", Harvard Business School Press, 2001.

Martínez, R. (2002). Balanced Scorecard: nueva metodología para el desarrollo de indicadores de gestión. Medellín, Universidad EAFIT. p. 85.

Pérez rivera, E. J. (2010). Implementación de un modelo Balanced Scorecard sustentado en el software BSC Designer. Tesis de Ingeniería Industrial. Universidad de Antioquia, p. 16.

Ramón-Jerónimo JM, et al. (2017) Utilidad del presupuesto y del cuadro de mando integral en la gestión de centros de atención primaria. Impacto sobre la motivación del personal. Aten Primaria.

Membrado Martínez J. (2013). Metodologías Avanzadas para la planificación y mejora. Editorial Diaz de Santos.

L'Huillier (2008), Gastón: IN77U.02 Control de Gestión, Universidad de Chile, Departamento de Ingeniería Industrial, MBA Puerto Montt.

Izaguirre Neira, J., \& Párraga Velásquez, M. (2017). Aplicación de las metodologías 8D y AMFE para reducir fallos en una fábrica de refrigeradoras. Industrial Data, 20 (2), 61-70. 